\title{
DOLL BASED DESIGN TOOL IN CORPORATE CONTEXTS: A QUALITATIVE COMPARISON WITH STORYBOARD IN A NEW PRODUCT DEVELOPMENT PROJECT
}

\author{
Kagohashi, Kaho (1); Taoka, Yuki (1); Ohno, Takehiko (2); Hamaguchi, Nana (2); Chen, Xinlei \\ (2); Amano, Kenta (2); Saito, Shigeki (3)
}

1: Tokyo Institute of Technology, School of Engineering, Department of Mechanical Engineering, Graduate Major of Engineering Design and Science; 2: NTT TechnoCross Corporation, ICT Design Office; 3: Tokyo Institute of Technology, School of Environment and Society, Department of Transdisciplinary Science and Engineering, Graduate Major of Engineering Design and Science

\begin{abstract}
Various tools for participatory design approach have been developed to support users to engage design process. Doll scenario is proposed as a generative tool for letting participants make and enact scenarios. However, suitable context to practice doll scenario is unclear in comparison with other tools using scenario. Therefore, our overall objective is to increase understanding of characteristics of making scenario in two different ways of expression; with doll and storyboard. We developed a doll scenario method, doll staging. The tool was evaluated in comparison to storyboard at a workshop which is a part of a new product development project in a corporate. The workshop was evaluated by semi-structured interviews with the participants and observations of the workshop and design outcome. The result suggests that doll staging allows participants to think from users' perspective in developing new idea. These findings provides new direction to choose scenario based design tools according to objective or context of design project. We also discuss potentials and research directions to use tools for developing scenario in corporate contexts.
\end{abstract}

Keywords: Participatory design, Doll scenario, Service design, Design methods

\section{Contact:}

Kagohashi, Kaho

Tokyo Institute of Technology

School of Engineering, Department of Mechanical Engineering

Japan

kagohashi.k.ab@m.titech.ac.jp

Cite this article: Kagohashi, K., Taoka, Y., Ohno, T., Hamaguchi, N., Chen, X., Amano, K., Saito, S. (2019) 'Doll Based Design Tool in Corporate Contexts: A Qualitative Comparison with Storyboard in a New Product Development Project', in Proceedings of the 22nd International Conference on Engineering Design (ICED19), Delft, The Netherlands, 5-8 August 2019. DOI:10.1017/dsi.2019.153 


\section{INTRODUCTION}

Participatory design approach has been researched as a way of involving users into design process as codesigners (Sanders and Stappers, 2008). Numerous number of tools have been proposed to help users to be creative without touching technical parts of design (Simonsen and Robertson, 2012). Some of the tools and techniques support users to express their tacit and latent knowledge by allowing them to make and enact scenarios which is story about people and their activities (Carroll, 1999; Simonsen and Robertson, 2012). Scenarios has been visualized through different ways such as storyboard, act-out and doll scenario (Simonsen and Robertson, 2012). Corporates are also interested in using scenario because involving users into their product or service development is becoming a competence for companies (Carli and Carli Lorenzini, 2017; Lettl, 2007). However, there are gaps between academia and design practice in corporate contexts, which hinder use of the tools: e.g. limited time for conducting design research (Visser et al., 2005). To our acknowledge, there are little understanding of what kind of expression of scenarios is appropriate for a particular purpose of each design workshop. Therefore, our objective is to increase understanding of ways of expressing scenarios in participatory design workshops. We conducted a twohour design workshop where storyboard and doll scenario were used to visualize ideas for new product development (NPD) in a Japanese corporation. As a preliminary analysis, this paper mainly reports characteristics of doll scenario.

\subsection{Participatory design methods}

As early participatory design projects aiming to incorporate worker opinions for improvement of their workspaces (van der Velden and Mörtberg, 2014), participatory design approach presumes equality, open discussion and commitments from participants (Yasuoka et al., 2013). Therefore, there have been a number of tools and techniques proposed in order to provide help to non-designers, who are not trained as designers, to be creative (Simonsen and Robertson, 2012). Among the tools, generative tools play a significant role in participatory design because participants can express their tacit and latent knowledge (Sanders, 2002; Witell et al., 2011), which shapes how people behave and what people needs in the future (Sanders, 2002). In generative sessions, participants engage design research through iterative three activities, make, tell and enact (Simonsen and Robertson, 2012). 'Tell' uses verbal language to describe future scenario of use. 'Enact' refers to use of the body in the environment to express ideas about future experience. 'Make' refers to use one's hands to embody ideas in the form of physical artefacts. Several types of generative tools have been proposed such as persona, contextmapping, 3D mock ups and scenariobased design (Sanders et al., 2010). Hansen reported potentials and challenges of using persona as a way of collecting users' insights in a participatory design workshop (Hansen, 2017). In the workshop, nondesigners created imaginal persona models based on their own experience. Imaginal persona models allow non-designers to express their own experience as the personas' experience rather than as their experience. Contextmpapping lets non-designers to map their context of interaction and emotion with products and/or services (Visser et al., 2005). As a scenario-based design tool, Brandt (Brandt and Grunnet, 2000) tested doll scenario, which allows non-designers to act upon "what if" scenario (Halse et al., 2010). Through enacting scenarios, the participants could tell what they think and dream.

\subsection{Ideation through scenario, doll scenario and storyboard}

Developing scenario has been considered as a powerful way of participatory design. Scenario enables rapid communication about usage of new services or products among different stakeholders (Rosson et al., 2002). Scenario has been expressed in different ways; words (Simonsen and Robertson, 2012), storyboard (Wikström and Verganti, 2013), doll scenario (Halse et al., 2010) and drama (Brandt and Grunnet, 2000). Storyboard is a short graphical depiction of a narrative (Truong et al., 2006). Design practice has been using storyboard as a way of visualizing users experiences of new products or services (Hanington and Martin, 2012). Storyboard enables professional designers to build empathy for end users, reframe experiences and consider design alternative (Hanington and Martin, 2012). Making storyboard encourages professional designers to consider situations and context of usage and feeling of intended users (van der Lelie, 2006). Currently, storyboard is getting to be used in participatory design (Wikström and Verganti, 2013). In participatory design, storyboard is creative process which helps people to shift the mind set from divergent to convergent (Andersson and Eriksson, 2011; Wikström and Verganti, 2013). Doll scenario 
is a way of letting non-designers to make and enact scenario with dolls. Doll scenario typically consists of several dolls and props which support visualization of scenes. Non-designers can immerse themselves into contexts and scenario of usage with making and acting doll scenario. Doll scenario has been used in different ways. Brandt (Brandt and Grunnet, 2000) reported a case where non-designers make stages with three backdrops to act current situations. Non-designers were asked to improvise scenario up to given "What if" scenario (Halse et al., 2010; Simonsen and Robertson, 2012). It helps non-designer to generate ideas. Doll scenario is also used as a way of experiencing new ideas (Jakobsen, 2012) and a way of building on proposed ideas (Yndigegn and Foverskov, 2011).

\section{RESEARCH QUESTION AND HYPOTHESIS}

Ideation through scenario is a powerful means of participatory design. Scenario can be expressed in different ways such as doll scenario and storyboard. To our acknowledge, it is still unclear how the expression styles impacts on non-designers in developing scenarios. Thus, there is no consensus about how designers can choose a way of expressing scenario. Our research objective is to increase understanding of characteristics of making doll scenario and of developing storyboard as a step to clarify how designers can choose scenario based design tools.

Our main hypotheses are that:

H1: Doll staging guarantees balanced participation of all participants. With Doll staging, it was expected that all participants contribute discussion by engaging 'make' activities together while lack of drawing skill prevents their participation to create storyboard.

H2: Doll staging provides new discoveries and ideas related to their scenario. Balanced participation is expected to bring participative discussion; thus participants can consider their scenario deeper with doll staging than storyboard.

\section{3 “DOLL STAGING", A TOOL FOR DOLL SCENAIO IN LIMITED TIME}

Based on previous studies (Halse et al., 2010; Yndigegn and Foverskov, 2011), we developed "Doll staging" to support creating a scenario through enacting with dolls. Doll staging aims to express a new concept which is typically an outcome of brainstorming in NPD. Duration of "Doll staging" was shortened from doll scenarios which had been proposed in order to fit limited time available in corporate contexts. Doll staging lasted for 30 minutes and has three components; three backdrops, dolls and props. The three backdrops represent current situation, ideas and happy endings. In first 10 to 15 minutes, participants are asked to determine a rough storyline and characters of the scenario and to create the backdrops and costumes of dolls with given materials. Duration of this phase was limited in order to avoid participants to create unnecessary details of backdrops, props and costumes. Otherwise, participants tend to be too enthusiastic at making props and backdrops. Then, participants are asked to move dolls by following rough storyline of their idea. It forces participants to enact, which allows participants to get new findings related to their scenario. Participants can stop acting whenever they find lacks of details or contradictions in their scenario. They are encouraged to create props which support acting the scenario and are able to change both the backdrops and costumes of the dolls. Participants are encouraged to iterate enacting the scenario. The materials and tools used in the workshop are shown in Figure 1. Pictures relevant to the design opportunity were mainly collected from the Internet. In addition, participants shared pictures of their surroundings prior to the workshop. Considering limited time for creation, the materials should be well selected. It would be better to prepare pre-made materials which enables to make costumes of dolls easily. If there are props which are likely to be made by participants, semi-completed materials, i.e. PC monitor, help participants to made props. The size of the backdrop is $30 * 40 \mathrm{~cm}$ and two picture were printed on a A4 paper.

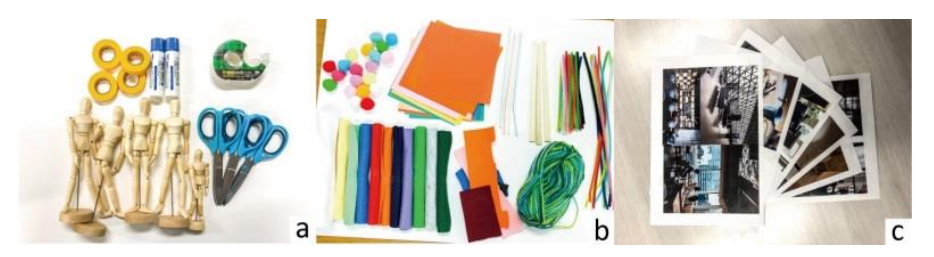

Figure 1. Materials prepared for doll staging 


\section{METHODS}

\subsection{Experimental design}

Doll staging and storyboard were compared in a design workshop which is a part of a NPD in a Japanese corporation. In the project, an employee, product owner (PO), has been developing a new product for supporting communication at remote work environments, where employees work together at different geographical locations, i.e. home and office. The objective of the workshop for the NPD is to increase understanding of how people communicate in the current remote work environments and of what hinders their experience of remote work. The workshop had eight participants who have experiences of remote work in the corporation. They do not work for design department but in several departments, i.e. engineering and marketing. In other words, they do not have prior experience of using doll and storyboard in their professional work. Therefore, they are considered as potential users of the new product and non-designers rather than as professional designers. The participants were divided into two groups of four participants depending on their motivation of remote work; either raising children at home or willingness to work effectively. Each group was facilitated by two authors and the workshop was facilitated by another author. The grouping and participants profile are summarized in Table 1. Group A consists of four employees working in the same department.

Table 1. Participants profile

\begin{tabular}{|l|l|l|l|}
\hline Group A & Willingness to work effectively & Group B & Raising children \\
\hline A1 & Business incubator (director), male 50s & B1 & Engineer, male 40s \\
\hline A2 & Business incubator, female, 30s. & B2 & Marketing, female 40s \\
\hline A3 & Business incubator (chief), female, 30s & B3 & Business operator, male 30s \\
\hline A4 & Business incubator (chief), female, 50s & B4 & Public Relations, female 40s \\
\hline
\end{tabular}

\subsection{Workshop procedure}

The 2-hour design workshop has mainly three design tasks which are (1) finding two design opportunity in remote work environments and (2) creating a scenario with doll staging and (3) creating a scenario with storyboard. The two ways of expressing scenarios were given in counterbalanced order.

Prior to the workshop, the participants were asked to write down three problems in their own experience of remote work and bring a picture of their remote work environment at their home. The first step of the workshop was to define two design opportunity in 10 minutes. The facilitator helped the participants to discuss two major problems based on the problems they thought about beforehand. The participants can expand their discussion beyond the problems they brought to the workshops. As a second and a third task, the participants were asked to ideate and visualize their service concept in 45 minutes in either doll staging or storyboard (Figure 2-a,b). The design opportunity of each design task was different between each other because the participants were asked to create a scenario for a design opportunity which they defined in the previous step. Examples of design outcomes of both of the tools were introduced to the participants prior to the design tasks in order to give overall understanding of the workshop. The examples are irrelevant to workshop theme, which were shown with either a video clip (doll staging) or a series of pictures (storyboard). After 45-minutes make, the participants presented their scenario of the service with their outcome. This presentation was video-recorded and not shared with the other group. As a wrap up of the workshop, both of the groups enacted their doll scenario in front of the other group, PO and the facilitators. A quick question and answer session followed to each enactment.

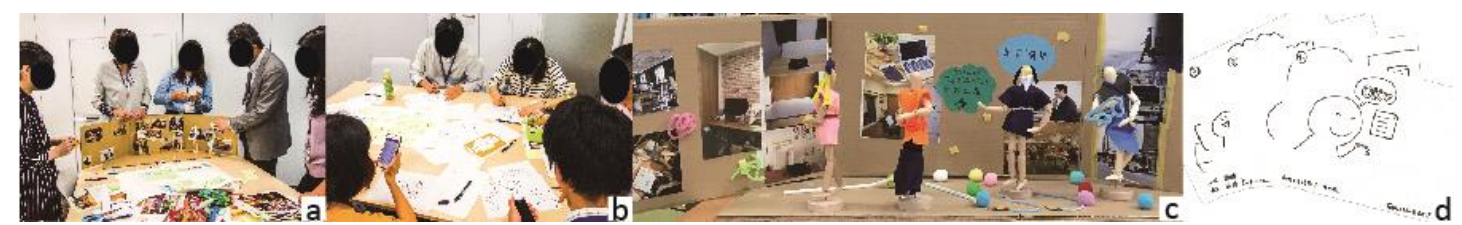

Figure 2. Looks in the workshop and outcomes 
Each 45-minutes design task consists of two steps; (1) idea generation and selection in 15 minutes and (2) scenario creation in 30 minutes. In the first 10 minutes, the participants were asked to generate service ideas related to remote work with sticky notes and to select one idea for further development. In the following 30 minutes, the participants engage on creating a scenario showing the new experience. In both condition of doll staging and storyboard, the participants had access to sticky notes, pens and big papers. Pictures showing remote work environments which were collected from the participants and from the Internet were also accessible in both of the condition. As a material to write down storyboard, A4 papers were given to the participants. Design outcome of the session are shown in Figure 2-c, d.

\subsection{Data collection and analysis}

The workshop was analysed by two methods; analysis of video recordings of both the workshop and design outcome and semi-structured interview with both the participants and PO. The video was recorded throughout the workshop to analyse differences in the participants' behaviour. As a preliminary analysis, we quote transcribed conversations and report observations by the authors. The semi-structured interviews were conducted by the authors for 30 minutes. It aims at evaluating perception of the participants to each design tool. Groups of two participants were interviewed by authors in a face-to-face setup at a time. We also had an interview with the PO for 30 minutes to explore potential and challenges of using Doll staging in corporate contexts. We also report transcribed quotes from the interviewees. In the result section, A1 refer to a participant in group A (cf. Table 1).

\section{RESULT}

Figure 3 shows timelines of how the participants engaged with Doll staging and storyboard. With Doll staging, both of the groups started to cut pictures for the backdrops with talking right after they started to create the scenario. On the other hand, with storyboard, the participants discussed their scenario without drawing. Making storyboard seemed to be separated into discussion and drawing phase. The participants in group B discussed with their storyboard because they finished drawing as a result of having started drawing at an earlier timing.

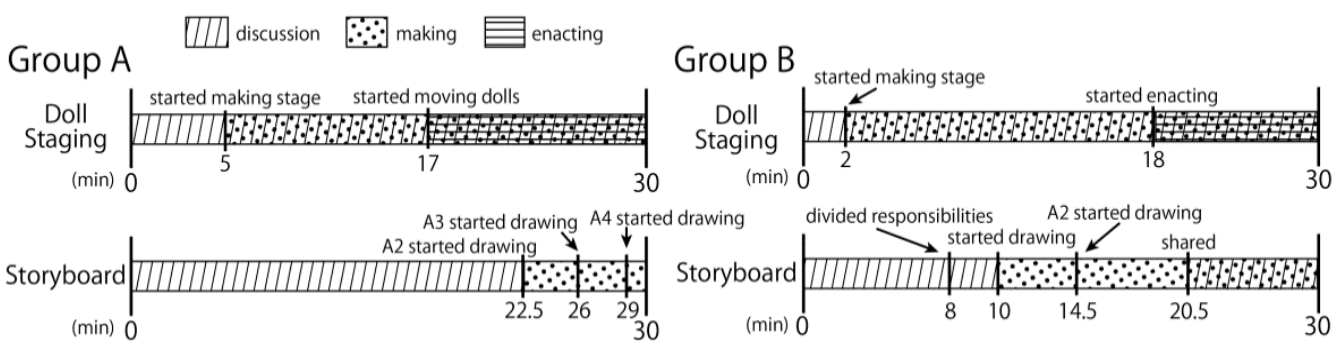

Figure 3. Participants' behaviours during the scenario creation

\subsection{Doll staging enables equal level of participation regardless capabilities of "make"}

Differences in participants' behaviours indicates that the degree of participation was more equal with Doll staging. The interview with the participants reveals participants perceive their participation in the same way. The results prove hypothesis $\mathrm{H} 1$.

\subsubsection{Participants behaviour during the scenario creation}

Participants commented difficulties of making materials for their scenario in both Doll staging and storyboard. We counted their utterances regarding negative reaction to making activities (e.g. "I cannot draw a chair. This is terrible, really terrible.") . The result was 7 times with storyboard and 3 times with Doll staging in group A, and 12 times with storyboard and 5 times with Doll staging in group B. It appears that they found less barriers of making materials to express their scenario with Doll staging.

The degree of participation was observed based on their "making" activities (cf. Figure3). In group A, the distribution of drawing storyboard was quite unbalanced. A1 (cf. Table 1) only discussed what to 
draw but wouldn't draw anything. A4 also hesitated to draw at most of the time and eventually did in last 1 minutes. Three out of four scenes were voluntary drawn by A2 and A3 because the participants recognized that A2 is good at drawing and A3 was the newest member within their department in the group. On the other hand, all of the participants of group A contributed to making activities in Doll staging. Similar tendency was observed in group B. They planned to create their storyboard which consisted of three scenes at first. Therefore, they assigned three participants (B1, B3, B4) to draw a scene. B2, who didn't have any allocated scene, had nothing to do for a while. After a facilitator proposed to make her be in charge of a new scene, she got her scene and started to draw. With doll staging, all of the group members naturally take part in preparing a stage and enacting with dolls throughout the time.

\subsubsection{Interview with the participants}

Participant A1 clearly mentioned equal participation among the group member with Doll staging:

Interviewer (I): "Are there any difference in teamwork between two tools, for example, equality of participation?"

A1: "[...] When we created a scenario with Doll staging, all of us contributed. On the other hand, I entrusted the drawing capability of A2 and A3 with storyboard. Looking back now, I don't feel that was good.[...] I hesitated to draw, like "I cannot draw anything!"."

I: "How was your hesitation with Doll staging?"

A1: "There was no hesitation with Doll staging. I could express something by just enacting with doll, but with drawing..."

His team member, A4 also stated the difference of the degree of participation. Their remarks show that their lack of confidence in drawing prevented their active participation with storyboard. In addition, both of them had negative feelings toward their unbalanced participation. B2 also mentioned the equal distribution of tools with doll staging as well as the participants of group A.

\subsection{Doll staging secures foundation for participative discussion}

The transcript of the workshop and the interview show several differences in what the participants found during creating scenarios. Furthermore, the participants emphasized that they had participative discussion with Doll staging in the interview. These results support our hypothesis H2.

\subsubsection{Participants behaviour during the scenario creation}

In group A, the participants seems to have noticed missing details related to their scenario with Doll staging. Questions were often raised, for example "Are they(characters) having a meeting here?" or "Ifeel this is strange.". As a result, they could have elaborated their scenario in response to their questions. There was no great difference in deepness of discussion in Group B. In group B, the participants could have elaborated their service by adding new ideas with both Doll staging and storyboard.

\subsubsection{Interview with the participants}

A4 and B4 pointed out the difference in their ways of discussion. Both of them perceived that it was easier to ask questions during Doll staging while they hardly ask questions during storyboard.

B4: I felt everyone was making the one thing during Doll staging. On the contrary, with storyboard, I felt I had to complete my own responsibility on my (allocated) scene. So, I got a little panic when I wondered what to draw next (in my scene).

A4: Doll staging seems better because we could discuss and make at the same time. [...] (With storyboard) When I come up a question about the scenario, we already divided the work so the discussion was...

I: Your members already started to draw.

A4: What is more, the illustration cannot be erased once you draw.

B1 and B2 explained that they could deepen their scenario more concretely with Doll staging. A3 mentioned how "enacting" made her be aware of the discrepancy in the group.

A3: It was not until we actually move dolls that I realized what other members thought was different from what I did. 


\subsection{Doll staging stimulated discussion about emotions of characters}

Surprisingly, scenarios which the participants generated were particularly different depending on ways of expression. Both of the groups focused on emotional aspects of characters with Doll staging while they explained technical details of their services in their storyboard. This difference was identified by the design outcomes, video observation, video transcript and interview.

\subsubsection{Design Outcomes}

All scenarios created with both storyboard and Doll staging had a similar storyline which consisted of three scenes: (1) a main character who is a user of their solution faced with a problem, (2) the participants' new service was introduced to solve the problem and (3) he/she felt happy thanks to the service. We found a difference of how the participants expressed their idea at second scene between storyboard and Doll staging.

In storyboard of both groups, a main character disappeared from their storyboard. The participants focused on explaining the service itself in detail, for example what technology enabled their service. Moreover, multiple functions were included with their storyboard. In group B, one of the functions was suddenly explained, although the problem which the function solves was not mentioned in the first scene.

On the other hand, with Doll staging of both groups, the participants focused on user's interactions with their solution by telling from user's point of view. Therefore, the participants did not mention technical parts of the service but touchpoint of their service even though they discussed the service in detail during the scenario creation.

We also found that the participants gave presentations of scenarios in different ways depending on the tools. The presentation was performed as a puppet show by both of the groups in doll staging; each participant enacted as a character with little narration. On the contrary, the participants of the both groups described their scenario with storyboard in a similar way to that of giving a PowerPoint style presentation. Even if character's speech bubbles were written on storyboard, participants hardly acted the characters nor changed their voice as if the characters talked. The participants seemed to tell their scenario more objectively with storyboard.

\subsubsection{Participants behaviour during the scenario creation}

During their works with Doll staging, participants mentioned the feelings and the activities of the characters plenty of times ( 5 times in group A and 7 times in group B). They often gave expression as if they were really characters in their scenario, e.g. A1 said "Then I (a character) work here busily. Like busy, busy, so busy." and B4 said "I (a character) decide to bring my souvenir to the office. 'These remarks were made during discussion, not as character's line in the rehearsal. There were less character-subjective utterances in making storyboard (2 times in group A and 3times in group B).

\subsubsection{Interview with the participants}

B4 explained how she worked with Doll staging;

B4: [...] It was easy to put emotion into the scenario when we moved the dolls. Because they were dolls, we imagined their personalities, such as ways of speaking. [...] It was a good way to reflect feelings and emotion in a problem because we ourselves became characters of doll staging.

B3, who has an experience of making customer journey map, stated that doll staging was similar to customer journey map because both of them look at emotion of users. B1 also referred to Doll staging as a good way to think from user's perspective based on his experience;

B1: When we explain new service ideas to users, we have a tendency to focus on how great the system of the service is. But with doll, I could emphasize what users would get as a benefit through a new service, so I felt this was a good way.

\subsection{Doll staging stimulate discussion about design outcomes}

Interview with product owner (PO) pointed out the that Doll staging and storyboard are different in terms of how audience react to the scenario. She described that she tended to understand the scenarios of storyboard as reasonable because she arbitrarily supplemented an interval between scene to scene. 
On the other hand, Doll staging showed a scene as a series of movements, not as a still image. Thus, she stated that she could have questions to the scenarios because she found gaps between her assumption and what the participants actually showed with Doll staging. She also stated that active questions and answers session was a good way to improve the idea.

\section{DIscussion}

\subsection{Pros and cons of storyboard and Doll staging}

The characteristics of Doll staging was revealed in comparison with storyboard (Table 2). Easiness of having workshops with Doll staging is obviously inferior to that of storyboard. Doll staging needs more materials (cf. Figure 1) while preparing storyboard does not require specific materials.

The results suggest that willingness to participation of participants seems to be influenced by two factors: actual skill and confidence of 'make'. Developing storyboard necessarily needs skill of drawing, which is a barrier of participation for those who don't have the capability such as A1 and A4. On the other hand, Doll staging includes multiple tasks such as cutting pictures, making props and moving dolls. Therefore, participants don't have to engage all types of these 'make' activities. Each participant can choose ways of his/her contribution depending on what they can do and what they are comfortable to do. In addition, even if participants have 'good-enough' drawing skill of drawing in storyboard, they may hesitate to draw. It could be because they are not confident with their skills. We believe the lack of confidence is caused by the knowledge of what 'good' drawing is. With Doll staging, as the participants are not familiar with it, they feel lower pressure to make 'good' backdrops and props. In other words, the participants may become unwilling to engage 'make' activities once they realize gaps of make capabilities between participants.

As we observed and participants commented, Doll staging appeared to trigger discussion more often than storyboard. This could be because participants easily share their work-in-progress artefacts with doll staging. Since artefacts were three-dimensional, it was unchallenging for participants to grasp what other member was making. Casual reaction to group members' work brings lively atmosphere to group and give a chance to start discussion related to the scenario (van der Lelie, 2006). We could find a similar moment even in storyboard task by group B. The participants shared their drawings, which allows them to have discussed their idea with others' drawings. It leads to have drown new objects into their storyboard. These participative discussion may be caused by sharing participants' opinions. Doll staging seems to create platform to share opinions and to find what is missing in the scenario as Brandt and Grunnet reported with act-out(Brandt and Grunnet, 2000).

Table 2. Comparison between doll staging and storyboard

\begin{tabular}{|l|l|l|l|l|}
\hline & Easiness of having workshop & Make skill & Confidence of skill & Trigger discussion \\
\hline Doll & Less & better & better & better \\
\hline Storyboard & better & less & less & less \\
\hline
\end{tabular}

\subsection{Doll staging forces people to think from users' perspective}

The perspectives focused in scenarios were quite different depending on the tools (Figure 4-a). With storyboard, participants focused on simply describing their service idea itself in detail, rather than eliciting emotion although storyboard should be ideally drawn to elicit emotion of characters (Hanington and Martin, 2012). It suggests that it may have been difficult for the participants to continuously stand on users' point of view with storyboard because they didn't have much experiences of creating scenario with storyboard. This deviation from user's perspective may also lead participants to mix up several functions in one scenario in their storyboard. With Doll staging, participants performed their scenario as a drama by enacting with dolls. It helped the participants to think from user's perspective throughout their scenario (Figure 4-a). Doll was probably considered as not a fictitious character but participant's alter ego. This helps participants to be immersed in the situation where characters were placed in. Therefore, participants might feel the world of their scenario from characters' points of view with less cognitive effort. Doll staging seems to be helpful for non- 
designers in designing with empathy for user. We believe the difference leads to create more realistic scenario with enacting as Simonsen and Robertson (2012) reported.

Doll staging seems to be easier to involve even the audience who listen to the presentation besides participants who actually make the stage than storyboard does. When we use a metaphor of a route between a start point to a destination to a real use of product/service to, the presentation of scenario is telling a story of how users follow the route (Figure 4-b). With storyboard, the audience tends to supplement an interval between scene to scene (as shown by dotted line in Figure 4-b) because of each scene is shown as a still image: storyboard allows to imagine untold context. Moreover, the participants created the middle part of storyboard out of user's perspective in this workshop: like putting map on. Thus, we believe the audience, PO, consider the shown scenario as reasonable. On the other hand, Doll staging encourages the audience to participate in improvement scenario. The audience could notice the gap between what the doll staging shows and what they imagine thanks to the scenes shown as a series of movements and the coherence of user-perspective. This gap provides another point to consider and leads to make the scenario closer to real use of products or services. Therefore, doll staging probably enables to brush up a concept including various stakeholders, even if the stakeholders are not part of 'make' activities.

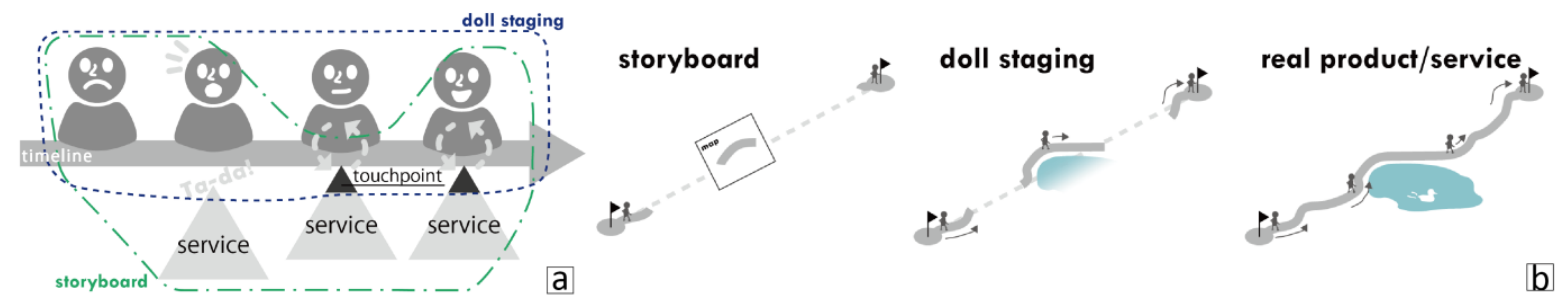

b

Figure 4. The differences in the way of telling/showing the scenario

\subsection{Potential and challenges of using doll staging in corporate contexts}

As this workshop was conducted as a part of new product development project in a company, we try to discuss potential and challenges of using doll scenario in companies. Doll staging successfully helps the participants to immerse themselves into a context of scenario, which help them to think emotion of characters in relatively limited time. Therefore, Doll staging has potential to be used in corporate context to address tacit and latent knowledge of users. In addition, doll staging can be used as a platform for discussion among professional designers and employees of other departments, i.e. engineer and sales, in companies because doll staging somehow forces people to think from users' perspective. As challenges, the current Doll staging focuses on visualizing a scenario. To demonstrate generated ideas for service, it would be also helpful to support generating and enacting multiple scenarios. Doll staging can be modified to this needs by, for example, making several stages in quicker and easier way. It is also important to find a way of recording discussion and ideas generated by participants during doll staging. Although their conversation during the workshop are valuable source of information (Visser et al., 2005), those comments are not necessarily directly reflected into a final scenario. In this experiment, the participants comments were not effectively recorded during the workshop. Therefore, video recordings need to be watched to capture the valuable information by facilitators after the workshop. It is time consuming and often not feasible in corporate contexts, where time for analysis is often limited (Visser et al., 2005). It is necessary to record the valuable information in a way which designers can review quickly.

We recommend that companies to use Doll staging in NPD, which can be used within their own company such as communication within a company so that they can know know-how of facilitating Doll staging and of customizing Doll staging into a particular context. As Doll staging can visualize interactions among several stakeholders along with timeline of use, Doll staging can be used in NPD which includes multiple stakeholders such as, communication tools and C-to-C platform.

\section{CONCLUSION}

In this study, we developed a doll scenario, Doll staging, as a way to express a scenario of service out of NPD. We explored characteristics of Doll staging in comparison to storyboard in a workshop of NPD. We found that Doll staging increases willingness of participation and triggers of discussion and 
forces participants, non-designers, to think from users' perspective. Therefore, we believe Doll staging can contribute to process of developing and refining a concept idea into a valid product or service in NPD in companies. For further development of Doll staging, it is recommended to make Doll stage which can record discussion easily. Future research will aim to establish clear guidelines to practice Doll staging in every situation with every participant.

\section{ACKNOWLEDGEMENT}

This research has been conducted in collaboration between Tokyo Institute of Technology and NTT TechnoCross Corporation. We appreciate the participants of the experiments.

\section{REFERENCES}

Andersson, J. and Eriksson, Y. (2011), "THE USE OF STORYBOARD TO CAPTURE EXPERIENCES", INTERNATIONAL CONFERENCE ON ENGINEERING DESIGN.

Brandt, E. and Grunnet, C. (2000), "Evoking the future: drama and props in user centered design", Proceedings of Participatory Design Conference PDC 2000, No. December, pp. 11-20.

Carli, G. and Carli Lorenzini, G. (2017), "USER INVOLVEMENT IN PHARMACEUTICAL PACKAGING DESIGN-A CASE STUDY”, 21ST INTERNATIONAL CONFERENCE ON ENGINEERING DESIGN, VANCOUVER.

Carroll, J.M. (1999), "Five reasons for scenario-based design", Proceedings of the 32nd Hawaii International Conference on System Sciences, pp. 1-11.

Halse, J., Brandt, E., Clark, B. and Binder, T. (2010), "Rehearsing the Future", The Danish Design School Press.

Hanington, B. and Martin, B. (2012), Universal Methods of Design: 100 Ways to Research Complex Problems, Develop Innovative Ideas, and Design Effective Solutions, Rockport Publishers.

Hansen, S.M.O. (2017), Designing an Interactive Installation with Sounds from Rural Areas Explorations of the Interactivity with Sounds.

Jakobsen, C. (2012), "Towards Doll based design - framework , guidelines and research potentials", Proceedings of the 7th Nordic Conference on Human-Computer Interaction: Making Sense Through Design, pp. 388-397.

van der Lelie, C. (2006), "The value of storyboards in the product design process", Personal and Ubiquitous Computing, Vol. 10 No. 2-3, pp. 159-162.

Lettl, C. (2007), "User involvement competence for radical innovation", Journal of Engineering and Technology Management - JET-M, Elsevier, Vol. 24 No. 1-2, pp. 53-75.

Rosson, M.B., Carroll, J.M., Tech, V. and Va, B. (2002), "Scenario-Based Design", Human-Computer Interaction, Boca Raton, FL, Lawrence Erlbaum Associates, pp. 1-35.

Sanders, E.B.-N. (2002), "From User-Centered to Participatory Design Approaches", in J. Frascara (Ed.), Design and the Social Sciences, Taylor \& Francis Books Limited.

Sanders, E.B.-N., Brandt, E. and Binder, T. (2010), "A framework for organizing the tools and techniques of participatory design", Proceedings of the 11th Biennial Participatory Design Conference on - PDC '10, ACM Press, New York, New York, USA, p. 195.

Sanders, E.B.-N. and Stappers, P.J. (2008), "Co-creation and the new landscapes of design", CoDesign, Taylor \& Francis, Vol. 4 No. 1, pp. 5-18.

Simonsen, J. and Robertson, T. (2012), Routledge International Handbook of Participatory Design, Routledge International Handbooks, Routledge, available at: https://doi.org/10.1177/0963662512444848.

Truong, K.N., Hayes, G.R. and Abowd, G.D. (2006), "Storyboarding: an empirical determination of best practices and effective guidelines", Proceedings of the 6th ACM Conference on Designing Interactive Systems - DIS '06, pp. 12-21.

van der Velden, M. and Mörtberg, C. (2014), "Participatory Design and Design for Values", Handbook of Ethics, Values, and Technological Design, pp. 1-22.

Visser, F.S., Stappers, P.J., van der Lugt, R. and Sanders, E.B.-N. (2005), "Contextmapping: experiences from practice", CoDesign, Vol. 1 No. 2, pp. 119-149.

Wikström, A. and Verganti, R. (2013), "Exploring storyboarding in pre-brief activities", Proceedings of the International Conference on Engineering Design, ICED, pp. 11-20.

Witell, L., Kristensson, P., Gustafsson, A. and Löfgren, M. (2011), "Idea generation: Customer co-creation versus traditional market research techniques", Journal of Service Management, Vol. 22 No. 2, pp. $140-159$.

Yasuoka, M., Nakatani, M. and Ohno, T. (2013), "Towards a culturally independent participatory design method: Fusing game elements into the design process", International Conference on Culture and Computing, Culture and Computing 2013, IEEE, pp. 92-97.

Yndigegn, S.L. and Foverskov, M. (2011), "Props to evoke 'the new' by staging the everyday into future scenarios", Seniorinteraktion.DkParticipatory Innovation Conference. 